ARTIGO

DE REVISÃO

\title{
Alguns aspectos básicos para uma proposta de taxionomia no estudo da composição corporal, com pressupostos em cineantropometria
}

\author{
Cláudia Cezar ${ }^{1}$
}

\section{RESUMO}

A antropometria é uma área de estudos essencial em cineantropometria, pois proporciona informações específicas sobre a análise da compleição física. As diferentes metodologias de análise da composição corporal estão classificadas como direta, indireta e duplamente indireta. A escolha metodológica deve estar diretamente relacionada com o nível de precisão de análise que se pretende obter: atômico, molecular, celular, tecidual ou genérico. Tais aspectos findam por especificar também o número de componentes da composição corporal a ser inferida (modelo de $2 \mathrm{ou}+$ componentes). A maioria das técnicas metodológicas visa encontrar a densidade corporal do(s) avaliado(s), para a partir daí, através de equações matemáticas, estimar o percentual de gordura corporal (GC). Os percentuais de GC em níveis saudáveis, do ponto de vista da saúde (segundo a Organização Mundial da Saúde) para sedentários ou praticantes regulares de exercícios físicos, devem ser maiores do que aqueles sugeridos para atletas. Com base nestes princípios, o peso ideal teórico deve deixar de ser sugerido, posto que a composição corporal é que influencia a saúde de forma relevante, não a massa corporal absoluta. Por fim, independente da metodologia utilizada, para se obter resultados confiáveis, válidos e fidedignos sobre a composição corporal faz-se necessário: identificar a população a ser avaliada; conhecer os custos, as limitações e as dificuldades técnicas de cada método; tornar-se proficiente, tanto do ponto de vista conceitual-teórico quanto práti-

1. Mestre em nutrição e especialista em fisiologia do exercício; Coordenadora do Núcleo de Estudos sobre Obesidade e Exercícios Físicos NEOBE/ CEPEUSP; Professora do Centro de Práticas Esportivas da USP (CEPEUSP).

Endereço para correspondência:

Cláudia Cezar

Pça. 2, R. Prof. Rubião Meira, 61

Cidade Universitária - Butantã

05508-900 - São Paulo, SP

E-mail: cezarc1@usp.br co, da técnica escolhida; definir as equações a serem utilizadas de acordo com a população a ser avaliada, principalmente para subgrupos específicos; observar atentamente a anamnese para oferecer explicação dos resultados de forma adequada e compreensível ao avaliado e, por fim, referenciar o resultado obtido com base em uma norma ou critério.

Palavras-chave: Antropometria. Análise corporal, metodologia. Cineantropometria.

\section{INTRODUÇÃO}

Para que o desempenho motor seja executado com êxito, tem-se por pressuposto que o indivíduo apresente boa desenvoltura em um certo número de capacidades motoras, tanto condicionantes (de âmbito quantitativo), quanto capacidades coordenativas (de âmbito qualitativo). A cineantropometria é a área da ciência que estuda o corpo humano de forma estática e dinâmica ${ }^{1,2}$. Em relação à estrutura, funções gerais e todas as suas relações internas e externas, com o objetivo principal de analisar o desempenho motor. E, neste sentido, especial atenção tem sido oferecida aos aspectos da aptidão motora, seguimentos do crescimento e desenvolvimento, além da análise de fatores intervenientes, inclusive os de ordem nutricionais e socioculturais ${ }^{1}$. De forma sintetizada e subjetiva, a palavra cineantropometria significa medida do homem em movimento ( $\mathrm{Ki}$ neanthropometry).

A antropometria, por etimologia, é o conjunto dos processos de mensuração do corpo humano, ou de suas partes. É, portanto, uma área de estudo essencial em cineantropometria, pois proporciona informações específicas sobre a análise da compleição física, como por exemplo a forma, a proporcionalidade e a composição corporal ${ }^{3}$. Como demonstrado em diversos estudos, a composição corporal interfere diretamente no desempenho motor, na capacidade de trabalho, assim como na saúde e, por conseqüência, na qualidade de vida dos seres humanos ${ }^{4-6}$. Devido justamente a tais aspectos, a composição corporal é uma das

Rev Bras Med Esporte - Vol. 6, № 5 - Set/ Out, 2000 


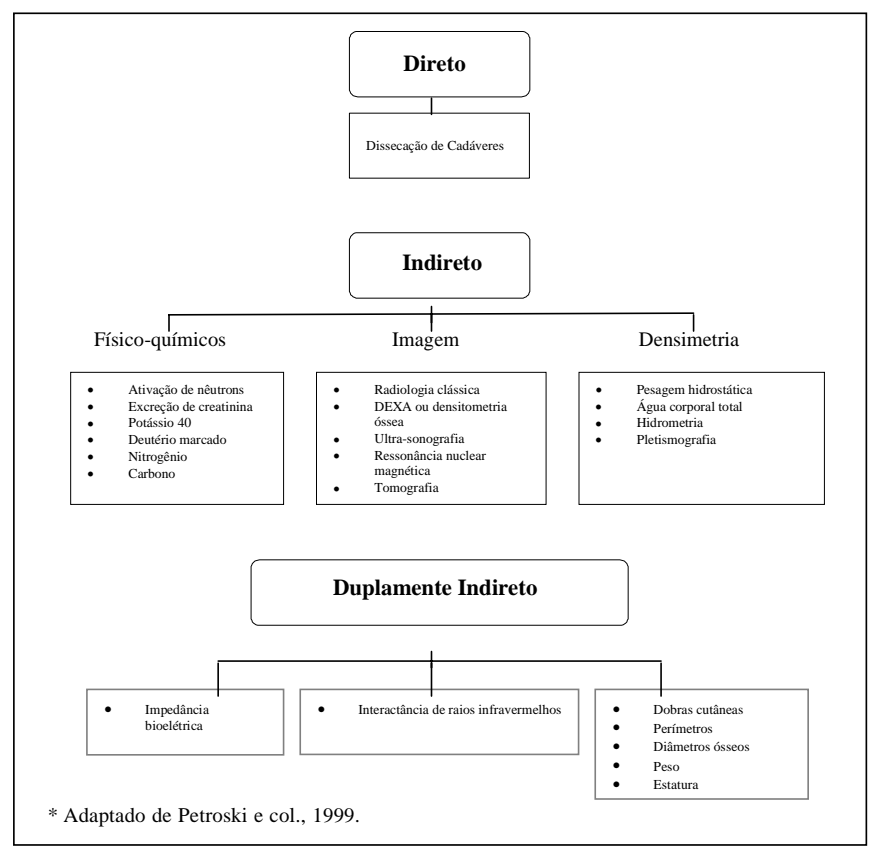

Fig. 1 - Classificação das metodologias de análise da composição corporal

áreas de grande interesse de pesquisa, seja em educação física, medicina desportiva, treinamento esportivo ou na área de nutrição.

A análise da composição corporal pode ser realizada através de uma série de metodologias amplamente descritas na literatura, bem como suas limitações e vantagens ${ }^{7-14}$. Algumas das técnicas estão citadas na figura 1.

\section{CLASSIFICAÇÃO DAS METODOLOGIAS DE ANÁLISE DA COMPOSIÇÃO CORPORAL}

Os métodos de análise da composição corporal são classificados como direto, indireto e duplamente indireto:

i) o método ser direto significa que os componentes teciduais foram avaliados diretamente, em separado, por autópsia ou dissecação de cadáveres.

ii) os métodos indiretos não oferecem tal oportunidade de manipulação e são assim considerados como métodos de análise da composição corporal in vivo. Em geral foram validados a partir do método direto e elaborados com base em métodos químicos e físicos, por imagem ou densitometria. Tais metodologias avaliaram fatores específicos que possibilitam, através de equações matemáticas, inferir sobre a densidade corporal do avaliado. O resultado de densidade obtido é aplicado a uma equação que estime a quantidade de gordura corporal (de Siri, por exemplo). Sabendo-se a GC, é possível estimar também a massa magra, absoluta ou percentual, do indivíduo. A maioria destes métodos está baseada, e validada, na técnica densitométrica. iii) os métodos duplamente indiretos também são técnicas para análise in vivo e, em geral, foram validados e correlacionados a partir dos resultados de métodos indiretos ${ }^{13}$.

$\mathrm{O}$ termo densitometria refere-se ao procedimento geral de estimar a composição corporal a partir da densidade corporal ${ }^{15}$. Este mesmo autor cita que as técnicas densitométricas buscam, na verdade, estimar o volume corporal, pois a massa dividida pelo volume resulta na densidade. A partir dos valores de densidade encontrados, calcula-se a percentagem de gordura corporal através da equação proposta por Siri ${ }^{16}$. Contudo, embora a densidade possa ser estimada com aceitável precisão na maioria dos grupos, os pressupostos para massa magra e de gordura, comumente utilizados para conferir densidade à composição, podem não ser válidos para muitos indivíduos ${ }^{14,15}$. A medida precisa de gordura corporal não é simples de ser realizada e continua a ser um significante desafio para a maioria das técnicas de composição corporal ${ }^{14}$.

\section{NÍVEIS E MODELOS DE ANÁLISE DA COMPOSIÇÃO CORPORAL}

Devido às diferentes técnicas e métodos de análise da composição corporal, os resultados encontrados não oferecem valores de gordura similares e freqüentemente são verificados em um nível e referidos, inadequadamente, em valores característicos de outro ${ }^{1}$. Pois como cada modelo de análise da composição corporal inclui mais de uma medida, há certa tendência de referir-se ao resultado como sendo analisado através de modelos fisiológicos ou celulares $^{14}$.

É possível, portanto, que a composição corporal seja referida de diferentes formas, devido aos distintos tipos de análise ou determinação dos variados tecidos. Wang et al. ${ }^{17}$ sugeriram que os métodos de análise da composição corporal podem ser organizados em cinco níveis, conforme mostrado na figura 2. Tais níveis estão amplamente discutidos na literatura, inclusive nacional ${ }^{10,11,18,19}$. A massa corporal pode também ser subdivida em compartimentos ${ }^{10}$. Estas subdivisões visam definir a operacionalização dos diferentes pressupostos teóricos e conceituais de metodologias de análise da composição corporal. Foram elaboradas com o objetivo de facilitar a utilização dos distintos procedimentos metodológicos e conferir, assim, maior ou menor validade a cada tipo de análise. A metodologia adequada ao trabalho que se pretende realizar deve ser avaliada em razão da precisão da composição corporal que se pretende obter.

O modelo clássico de dois componentes (2-C) da composição corporal divide o corpo em duas partes, das quais uma consiste em gordura corporal, e todos os tecidos remanescentes são reunidos e denominados como massa li- 


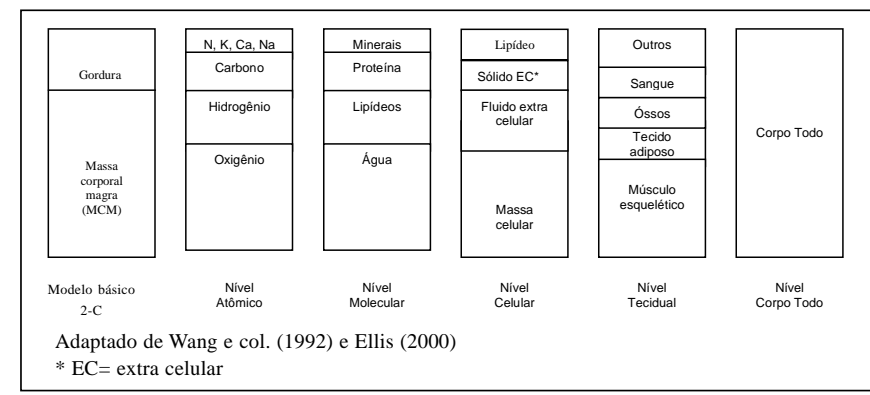

Fig. 2 -Modelo clássico de 2 componentes e de multicomponentes, em cinco níveis de análise da composição corporal

vre de gordura - $\mathrm{MLG}^{14}$. Em geral os métodos utilizados para analisar estes 2 componentes são aqueles que identificam a densidade corporal total, como pesagem hidrostática. Entretanto, a densidade para a massa livre de gordura para o modelo 2-C é uma constante assumida e, portanto, satisfatória para população específica analisada: adultos saudáveis ${ }^{14}$.

Devido às limitações do modelo 2-C, o modelo de 3 componentes (3-C) foi desenvolvido a fim de reduzi-las e, para tanto, incluiu-se a medida de água corporal total aos resultados obtidos pela pesagem hidrostática. Neste modelo, 3$\mathrm{C}$, a massa livre de gordura é dividida em 2 partes: conteúdo líquido (água) e sólidos remanescentes (predominantemente proteínas e minerais). Segundo Ellis ${ }^{14}$, para este método, os valores de densidade da água, gordura e sólidos corporais podem ser utilizados para adultos saudáveis, idosos e crianças. Mas não para pacientes com massa proteica, ou óssea, corporal significantemente depletada. Pois os valores de densidade dos compartimentos sólidos resultaria incorreta e a estimativa final de massa de gordura corporal seria também imprecisa.

Para desenvolver o modelo de 4 componentes, assumiuse que a densidade para a proteína corporal e os minerais ósseos são 1,34 e $3,075 \mathrm{~kg} / \mathrm{l}$, respectivamente ${ }^{20}$. A massa livre de gordura é dividida em três compartimentos básicos ou fisiológicos: massa celular corporal (MC), água ou fluido extracelular (FE) e sólidos extracelulares (SE). Assim a MIG é então definida como MC + FE + SE e a massa de gordura total como a MIG menos o peso corporal (massa). Uma das limitações deste modelo é que o erro de medida é cumulativo e transferido diretamente à estimativa final ${ }^{14}$.

O modelo multicomportamental está relacionado a ampliar o número de compartimentos analisados. No entanto, cada medida adicional deve ser independente das medidas anteriores e propostas para analisar compartimentos distintos ${ }^{10,14,19}$.

A análise de corpo todo tem por pressuposto utilizar justamente um único método, como por exemplo a pesagem hidrostática. Entretanto, quando somente um método é uti- lizado, as limitações técnicas resultam em maior imprecisão, associada à aquele método ${ }^{10,14,19}$.

\section{TERMINOLOGIA DOS DIFERENTES COMPONENTES DA COMPOSIÇÃO CORPORAL}

Após utilizar uma das metodologias disponíveis para quantificar a gordura corporal, o resultado obtido pode ser inferido como massa corporal magra ou como massa isenta de gordura. A diferente terminologia está relacionada aos diferentes métodos de análise das mesmas. A massa isenta de gordura (MIG ou fat free body-FFB) consiste de todo resíduo químico e tecidual do corpo. Inclui água, músculo, ossos, tecido conjuntivo e órgãos internos, menos todo tipo de lipídio, inclusive aquele da camada bilipídica das membranas celulares. Por outro lado, o termo massa corporal magra (MCM ou lean body mass-LBM) ou massa livre de gordura (fat free mass - FFM) inclui certa quantidade mínima de lipídios, aproximadamente 2 a $3 \%$ do total de lipídios nos homens e 5 a $8 \%$ nas mulheres. $\mathrm{O}$ tipo de análise de cada componente (MIG ou MCM) difere também, pois a MIG é analisada in vitro (a partir de solventes orgânicos) e a MCM in vivo ${ }^{21}$.

\section{PROPORÇÃO DE GORDURA CORPORAL PARA GARANTIR BOA CONDIÇÃO DE SAÚDE}

A proporção de gordura corporal (\%GC), em síntese, pode ser classificada como adequada (saudável), excessiva (estado de obesidade) ou reduzida (abaixo do necessário, condição de desnutrição), através da percentagem avaliada no organismo, a qual pode ser obtida pelo seguinte cálculo ${ }^{10}$ :

$$
\% \mathrm{GC}=\frac{\text { massa de gordura }(\mathrm{kg})}{\text { massa corporal total }(\mathrm{kg})} \times 100
$$

A recomendação para o percentual de gordura corporal, em relação ao peso absoluto (massa em $\mathrm{kg}$ ), embora não em um senso comum exato entre os especialistas da área, é apresentada na literatura em diferentes valores, os quais seguem próximos aos preconizados por Lohman ${ }^{21}$, descritos na tabela 1 .

TABELA 1

Percentagem de gordura corporal para homens e mulheres

\begin{tabular}{lcc}
\hline \multicolumn{1}{c}{ Níveis de GC } & Homens & Mulheres \\
Alto risco & $\geq 25 \%$ & $\geq 30 \%$ \\
Acima da média & $17-24 \%$ & $27-29 \%$ \\
Média & 14,5 a $16 \%$ & 22 a $27 \%$ \\
Abaixo da média & $6-14 \%$ & $12-22 \%$ \\
Alto risco & $\leq 5 \%$ & $\leq 12 \%$ \\
\hline
\end{tabular}

Adaptada de Lohman (1992) in Heyward and Stolarczyk, 1996, pg5.

a - alto risco para doencas e desordens associadas a obesidade

- alto risco para doenças e desordens associadas a desnutrição 
A obesidade proporciona sérios riscos à saúde, mas, por outro lado, níveis baixos de gordura corporal também potencializam danos consideráveis ${ }^{22}$. Está bem definido na literatura, inclusive nacional, que alta proporção de GC (obesidade) potencializa aumento na concentração plasmática de lipoproteínas de baixa densidade ( $L D L-C$ low density lipoprotein cholesterol), com concomitante redução na concentração sangüínea nas lipoproteínas de alta densidade (HDL-C high density lipoprotein cholesterol). Estas frações alteradas proporcionam a formação da placa de ateroma nos vasos sangüíneos, para culminar em enfarte ou acidente vascular cerebral.

Por outro lado, o organismo necessita de lipídios em quantidades mínimas (20 a 25\% do valor calórico total da dieta) para desenvolver as funções fisiológicas essenciais. Como, por exemplo, a formação de membranas celulares, fornecimento energético, transporte e estoque de vitaminas lipossolúveis (A, D, E e K). Os lipídios também estão envolvidos no funcionamento dos sistemas nervoso e reprodutor, no ciclo menstrual, assim como no crescimento e maturação sexual durante a pubescência ${ }^{10,23}$.

Os indivíduos que apresentam reduzida proporção de gordura corporal freqüentemente são portadores de desordens alimentares, como, por exemplo, desnutrição. Tais sujeitos são facilmente encontrados em grupos de bailarinas e modelos (fotográficos ou de passarela), além de atletas de algumas modalidades esportivas. Embora estes grupos sejam positivamente evidenciados pela mídia, não podem ser considerados como parâmetros de saúde. Esta condição subestima e banaliza a sugestão de valores percentuais adequados de gordura corporal, do ponto de vista da saúde. Os valores apresentados na tabela 1 estão de acordo com os valores propostos pela Organização Mundial da Saúde $^{24}$, justamente porque tais percentuais de gordura corporal não trazem danos à saúde. Entretanto, com frequiência pessoas que passam a participar de atividades físicas em academias de ginástica são orientadas para apresentar percentuais de gordura corporal extremamente inferiores àqueles considerados saudáveis. Tais informações estimulam tanto o aluno sedentário, como o praticante regular, ou irregular, de exercícios físicos, a apresentar (ou no mínimo buscar), reduzir severamente seu percentual de gordura corporal. Esta prática potencializa comportamentos alimentares inadequados para que tais metas sejam atingidas. Estas condições de reduzida concentração de GC podem eventualmente induzir consequiências dramáticas, como gerar anemia profunda (a qual eventualmente não tem retorno) ou anorexia.

Neste sentido, os indivíduos que profissionalmente têm como prática manter valores de gordura corporal extremamente reduzidos devem ser orientados a não permanece- rem por períodos prolongados numa condição limítrofe, para evitar assim o desenvolvimento de anemias, desnutrição ou anorexia, além de outros sérios danos bioquímicos e fisiológicos ao organismo. Mas em pior situação fica o aluno de academia que não tem tais aspirações profissionais, mas sente-se estimulado a manter permanente para si tais valores restritivos de gordura corporal. Estes indivíduos são os alvos mais frágeis das sérias conseqüências citadas.

\section{ÍNDICES CORPORAIS PARA SUGESTÃO DA CONDIÇÃO DE SAÚDE}

Entre os diversos índices corporais, o índice de massa corporal (IMC), de Quetelet ${ }^{25}$ é um dos mais utilizados. Entretanto, este índice não leva em consideração a exata composição corporal do indivíduo. Não é um indicador de proporção de gordura corporal. Portanto é inadequado para inferir com precisão sobre o percentual de gordura corporal do avaliado, pois pode conduzir a conclusões inadequadas (sub ou superestimadas).

Por outro lado, para estudos epidemiológicos o IMC pode ser utilizado como indicador de estado de saúde, como desnutrição e obesidade. O IMC é justamente utilizado quando da falta de melhores recursos, técnicos ou financeiros, para o mesmo fim ${ }^{24}$. Como outro exemplo, o IMC é um ótimo indicador para diagnosticar e acompanhar a condição de saúde de escolares, que pode ser realizada através da antropometria bianual realizada pelos professores de educação física na escola ${ }^{26}$.

\section{PESO IDEAL: UM MITO IRREAL}

O peso ideal, segundo De Rose et al. ${ }^{3}$, pode ser obtido através do cálculo:

$$
\text { Peso ideal }=\frac{\text { massa corporal magra }}{1-\frac{(\% \text { gordura ideal })}{100}}
$$

Para obter-se o valor para a massa corporal magra é preciso subtrair a gordura absoluta $(\mathrm{kg})$ do peso corporal (massa), também em kg.

Massa corporal magra $=$ peso corporal - gordura absoluta

Para calcular gordura absoluta:

Gordura absoluta $=$ peso $($ massa $) \mathrm{kg} \mathrm{x} \frac{(\% \text { gordura corporal })}{100}$

O percentual de gordura corporal pode ser obtido pela expressão matemática de $\operatorname{Siri}^{16}$ : 


$$
\% \mathrm{GC}=\left[\frac{4,95}{\text { densidade }\left(\mathrm{g} / \mathrm{cm}^{3}\right) \text { do avaliado }}-4,50\right] \times 100
$$

\section{DEFINIÇÃO DA PROPORÇÃO DE GORDURA CORPORAL "IDEAL"}

O peso ideal, quando sugerido, deve ser feito pelo menos sob uma certa faixa de amplitude (mínima e máxima). Por exemplo, os percentuais mínimo e máximo de gordura corporal indicados para a faixa de idade e sexo, sugeridos na tabela 1 , conforme adaptação da expressão matemática abaixo:

$$
\begin{aligned}
& \text { Peso ideal mínimo }=\frac{\text { massa magra }}{1-\frac{(\% \text { gordura ideal mínima })}{100}} \\
& \text { Peso ideal máximo }=\frac{\text { massa magra }}{1-\frac{(\% \text { gordura ideal máxima })}{100}} \\
& \text { de acordo com a idade }
\end{aligned}
$$

Neste sentido, o peso ideal não deve ser oferecido como um valor fixo, por exemplo 59kg. Na maioria dos casos é de bom senso nem evidenciá-lo, justamente por ser apresentado como massa absoluta $(\mathrm{kg})$ e não em percentual de gordura corporal e massa magra.

Outro aspecto negativo de trabalhar com sugestão para peso ideal é que tal valor não é constante, principalmente para a mulher, devido à retenção hídrica, pertinente ao ciclo menstrual. Outro fator importante quanto à sugestão de peso ideal é que o envelhecimento proporciona acúmulo fisiológico de gordura corporal e, portanto, caso o indivíduo consiga manter-se no mesmo peso (massa) de décadas atrás, não significa que a proporção dos componentes corporais seja a mesma. Em geral, o processo de envelhecimento, quando não há realização de exercícios físicos regulares, proporciona redução fisiológica de massa muscular e óssea ${ }^{23}$.

\section{CONCLUSÃO}

Pelo que foi discutido acima, independente da metodologia utilizada, para que o resultado de análise da composição corporal seja objetivo, válido e confiável, faz-se necessário identificar alguns aspectos básicos:

1) A idade, sexo, etnia, estimativa de gordura corporal (obesos/desnutridos) e nível de condicionamento físico dos avaliados são os principais fatores que devem ser levados em consideração para a escolha da metodologia de análise da composição corporal, além de verificar se a população a ser avaliada aceita, ou tem as características necessárias, para submeter-se ao tipo de avaliação a ser proposta;

2) Outro fator preponderante para definir o método adequado de avaliação da composição corporal é conhecer os custos, as limitações e as dificuldades técnicas de cada metodologia;

3) Após a decisão de qual método utilizar, é preciso tornar-se proficiente, tanto do ponto de vista conceitual-teórico quanto prático, da técnica escolhida, a fim de diminuir uma das principais fontes de erro metodológico;

4) É preciso também definir as equações a serem utilizadas, principalmente para subgrupos populacionais específicos, para poder proporcionar maior precisão na avaliação da composição corporal do avaliado;

5) A anamnese e o histórico esportivo do avaliado devem ser observados atentamente para que o resultado da avaliação não frustre o indivíduo. (Um exemplo é o caso de alguém que, embora apresente alto percentual de gordura corporal, esteja com valores significativamente menores em relação à avaliação anterior, justamente devido a estar sob orientação para mudança do comportamento alimentar e motor. Em geral, o avaliador desavisado desmotiva, ou até mesmo frustra, um indivíduo obeso que está ativo e disciplinado, um dos principais fatores dos alunos não retornarem para a avaliação física, da qual a composição corporal é apenas um dos componentes).

6) Para oferecer informações precisas sobre a composição corporal faz-se necessário ter uma perspectiva de referência. Uma referência pode ser estar baseada em normas, como subgrupos claramente definidos (homens, mulheres, faixa etária, condição de treinamento ou modalidade esportiva, etc.). Estes resultados baseados em normas podem também ser os valores do próprio grupo, por exemplo, pré e pós alguma intervenção. Ou a perspectiva de referência pode estar baseada em um critério, como, por exemplo, valores predeterminados ${ }^{27}$.

\section{AGRADECIMENTOS}

A elaboração deste material somente se tornou possível graças ao apoio do Prof. Dr. Pio Colepicollo, do Instituto de Bioquímica da USP, e dos diretores Edison Baccani e João Carlos Kanaãn, do Centro de Práticas Desportivas da USP.

\section{REFERÊNCIAS}

1. Kiss MAPD, Böhme MTS, Regazzini M. Cineantropometria. In: Ghorayeb N \& Barros T. O exercício. São Paulo: Atheneu, 1999: 117-30.

2. Beunen G, Borms J. Cineantropometria: raízes, desenvolvimento e futuro. Revista Brasileira de Ciência e Movimento 1990;4;76-97.

3. De Rose EH, Pigatto E, De Rose RC. Cineantropometria, ed. física e treinamento desportivo, Rio de Janeiro: SEDE/MEC, 1984. 
4. Forbes GB. Exercise and lean weight: the influence of body weight. Nutrit Rev 1992;50:157-61.

5. Bouchard C, Shephard RJ, Stephens T. Physical activity, fitness, and health. Human Kinetics 1994:1-1040.

6. Flatt JP. Integration of the Overall Response to Exercise. Int J Obes 1995; 4:S31-S40.

7. Lohman TG, Roche A, Martorell R. Anthropometric standartization reference manual. Champaign, IL.: Human Kinetics, 1988.

8. Heyward VH. Advanced fitness assessment and exercise prescription. Champaign - Ilinois: Human Kinetics, 1991.

9. Malina RM, Bouchard C. Models and methods for studying body composition In: Malina, R.M \& Bouchard, C. Growth, maturation and physical activity. Champaign, IL.: Human Kinetics, 1991.

10. Heyward VH, Stolarczyk LM. Applied body composition assessment. Champaign, IL.: Human Kinetics, 1996.

11. Roche AF, Heymsfield SB, Lohman TG. Human body composition. Champaign, IL.: Human Kinetics, 1996.

12. Petroski EL. Antropometria, técnicas e padronizações, Porto Alegre: Palotti, 1999.

13. Costa RF. Avaliação da Composição corporal - CD-Rom, Phorte Editora, São Paulo, 1999.

14. Ellis KJ. Human body composition: in vivo methods. Physiol Rev 2000; 80:649-80.

15. Going SB. Densitometry. In: Roche et al. Human Body Composition Champaign, IL.: Human Kinetics, 1996.

16. Siri WE. Body composition from fluids spaces and density: analysis of methods. In: Brozek J, Henschel, A. Technics for measuring body composition. Washington D.C.; Nat. Acad. Of Scienc. Nat. Res. Council, 1961. 223-44.
17. Wang ZM, Pierson RN Jr, Heymsfield SB. The five-level model: a new approach to organizing body-composition research. Am J Clin Nutr 1992; 56:19-28.

18. McArdle WD, Katch FI, Katch VL. Fisiologia do Exercício, New York, Guanabara/Koogan, 1996:513-661.

19. Guedes DP, Guedes JERP. Controle do peso corporal. Paraná: Mediograf, 1998.

20. Snyder WS, Cook MJ, Nasset ES, Karhausen LR, Howells GP, Tipton IH. Report of Task Group on Reference Man: ICRP-23. New York: Pergamon, 1984. In: Ellis KJ. Human body composition: in vivo methods. Physiol Rev 2000;80:649-80.

21. Lohman TG. Advances in body composition assessment. Current issues in exercise science series. Monograph (1992) In: Heyward VH, Stolarczyk LM. Applied body composition assessment. Champaign, IL.: Human Kinetics, 1996.

22. Newsholme EA, Leech AR. Catabolism of lipids. In: Biochemistry for the medical sciences, New York: Lea \& Sons, 1983.

23. Wilmore JH, Costill DL. Physiology of sport and exercise. Human Kinetics, 1994:124-45.

24. World Health Organization. Antropometry as an indicator of nutritional and health status. In Physical status: the use and interpretation of anthropometry. Geneva, 1995.

25. Must A, Dallal GE, Dietz WH. Reference data for obesity: 85th and 95th percentiles of body mass index $\left(\mathrm{wt} / \mathrm{ht}^{2}\right)$ and triceps skinfold thickness. Am J Cin Nutr 1991;53: 839-46.

26. Cezar C, Teixeira LR. Diagnóstico e prevenção da obesidade: o professor de educação física pode atuar como agente de saúde na escola. Rev Paul Med, 2000 (no prelo).

27. Morrow JR, Jackson AW, Disch JG, Mood Dale P. Measurement and evaluation in human performance. Champaign, IL.: Human Kinetics, 1995. 